\title{
"Hollow Heart Disease" Among Contemporary Art Students in College and Ways to Tackle it
}

\author{
${ }^{*}$ Xiao Mengyi ${ }^{1}$, Zeng Sicheng ${ }^{2}$, Yang Ming $^{3}$
}

\author{
${ }^{1}$ Chengdu University, Chengdu, Sichuan, 610100 \\ ${ }^{2}$ Chengdu University, Chengdu, Sichuan, 610100 \\ ${ }^{3}$ Chengdu University, Chengdu, Sichuan, 610100 \\ *Corresponding author. Email: 2898481428@qq.com
}

\begin{abstract}
As time rapidly advances, the mental features of college art students are also under constant changes. A variety of mental diseases emerge one after another, which has become an urgent concern for colleges and universities. Strengthening mental health education for art students is an important measure to comprehensively implement the Party's educational policy and promote quality education under the new situation, an important task to strengthen students' ideological and political education, and an important way to train healthy, optimistic and high-quality applied art talents. Under this research background, the author of this paper psychologically evaluated college art students through a questionnaire survey. The results showed that the "hollow heart disease" was prevalent among the responding students, and the disease had become one of the major mental diseases affecting college art students. In this paper, aiming at the problem of " hollow heart disease" of art students, an in-depth study is carried out to study the current situation of mental health education for art students. Summarized eight symptoms of "hollow heart disease" of art college students, the main reason and uniqueness of its formation. Put forward three effective ways to get rid of the "hollow heart disease" of art college students, providing data support for cultivating more artistic talents with three views, goals and professional qualities.
\end{abstract}

Keywords: art students in college, "hollow heart disease", mental health education

\section{INTRODUCTION}

With the rapid development of China's economic construction, the success of building a well-off society in an all-round way has provided college students with unprecedented material conditions, comfortable living security and diversified learning resources. However, the prosperous life makes some college students appear some abnormal phenomena, such as some students are filled with extreme emptiness and don't know the value and goal of life. In response to this phenomenon, Xu Kaiwen, deputy director of the Center for Mental Health Education and Consultation of Peking University, pointed out in his speech entitled "The Economics of Hollow Heart Diseases and Anxiety in the Times" published in April 2017 that in recent years, some college students in China are experiencing, experiencing and suffering from a mental health problem caused by deviation or lack of ideals and beliefs and value orientation [1]. Although its symptoms are similar to depression, due to the lack of clear diagnosis results and evidence from relevant scales, it is defined as "mental disorder caused by value defects", Commonly known as "hollow heart disease".

Art college students are a special group of contemporary college students. Like ordinary college students, they are full of vigor, rich feelings, ideals and pursuits. However, influenced by professional nature and personality characteristics, it has certain particularity, such as emotional sensitivity and emotional volatility; The idea is more unconstrained, creative and perfectionist; I like to be alone and not talk well in communication; Comprehension and self-discipline are lower than those of ordinary students [2]. Compared with ordinary college students, these characteristics are easier to enter the "hollow heart disease" army and become one of their important psychological diseases. It has become an urgent task for art talents education in colleges and universities to study the causes and characteristics of the "hollow heart disease" of art students and put forward relevant solutions. 
The symptoms of "hollow heart disease" are similar to mild depression, and only show loneliness and emptiness in the early stage, without life worries. However, long-term patients with "hollow heart disease"will gradually appear self-loathing, lack of value recognition and other phenomena, and even worse, they will have suicidal thoughts, which will lead to other serious mental diseases. Therefore, in order to prevent the frequent occurrence of "hollow heart disease" among art college students, it is necessary to fully consider the prevailing psychological conditions and characteristics of contemporary college students, and pay attention to understanding their personality characteristics, family characteristics, learning characteristics, psychological characteristics, specific analysis of specific characteristics, and targeted guidance by classification. Only in this way can we avoid other psychological problems caused by "hollow heart disease" of art students, cultivate art talents with three views, high artistic attainments and mental health, and contribute to the improvement of people's spiritual life world.

\section{METHOD}

\subsection{Questionnaire survey}

To better keep tabs on the mental health conditions of college art students, we should carry out mental health education as soon as the students set foot on the campus. Mental files should be compiled, psychological questionnaires should be conducted, and mental health lectures and interviews should be organized. An online questionnaire was distributed two months after the freshmen enrolled. A total of 25 questions were designed, including questions like "Have you set a clear goal for your future?" and "Are you feeling optimistic about your future?" We distributed 826 questionnaires inside the campus, of which $658(80 \%)$ retrieved questionnaires turned out to be valid. Investigation into the valid samples showed currently among the responding students, there are 204 "hollow heart disease" sufferers, accounting for $31 \%$ of the surveyed sample, indicating that the disease might have become one of the most common mental health conditions for college students.

\subsection{Analysis of survey results}

The results of the questionnaire survey showed that the hollow heart disease among college art students has the following eight manifestations: 1) Depression: diagnostically similar to the symptoms of depression, with diminished interest, bad moods, and failure to feel pleasure; 2) Loneliness: a strong sense of loneliness and meaninglessness; 3) Self-deficiency: lack of values supporting the student' $\mathrm{s}$ meaningful existence, with early symptoms of confusion and self-identity issues; 4) External recognition: desire for good interpersonal relationships, as manifested in the students' constant efforts in maintaining a good self-image in front of others; 5) Fear of being judged: exhibiting a strong aversion and fear toward being judged, with secondary manifestations of aversions to study and examinations; 6) Self-hatred: out of the thought that life and learning are meaningless, the students think they are not doing something meaningful and valuable, or they don' $t$ think the things are worth doing or against their conscience, so they hate themselves to the extent that they even want to commit suicide; 7) Suicidal thoughts: strong will to commit suicide, but they don' t really want to end their own lives. Instead, they just don' t know why they should live. They tend to try milder and less painful ways of committing suicide. 8) Course of disease: suffering from the disease for a long time (over a year), insensitive to biotherapy with poor performance of traditional psychotherapy.

\subsection{Analysis the Reasons for "Hollow Heart Disease" among College Art Students}

\subsubsection{College students' natal family enables the "hollow heart disease"}

Most college art students were born in a rich family. Some of their parents are believers of the so-called exquisite egoism[3]. They are busy with their own careers and tend to neglect their children. With defective education philosophies, they don' t spend enough time with their children, and instead, what parents do is to give children more money to satisfy their material needs. Spoiling like this may result in the stubborn personality of children. Children tend to be more emotional and go to extremes. They are more self-centered with weaker interpersonal skills. As time gradually passes by, issues like social withdrawal and dormitory conflicts might arise. On such occasions, with low self-control and selfconditioning abilities, these students cannot see things in a bigger picture. Without necessary guidance, various psychological problems may arise among them. Besides, children who grow up in such families tend to exhibit values straying from China' s core socialist values or lack of values, the willingness to work hard, specific goals in life, and the senses of happiness and accomplishment through hard-working, all of which have contributed to the existence of "hollow heart disease."

\subsubsection{The special education art students receive fails to intrigue students' interests}

The traditional exam-oriented education in China also has an important impact on the student' $s$ mental health As the Chinese saying goes: "Everything is about the score." Teachers tend to overlook or even neglect the cultivation of students' morality, aesthetic judgment and interests. Under the dual pressure of exams and college entrance, some high school students who were 
scheduled to take the normal college entrance exam but may not get enough scores to get into college, will enroll in some training camps to equip themselves with art skills so that they can get extra scores from artistic exams. The fact is, these students only attend crash courses in a specific art discipline, which means some of them may not actually like their majors, let alone to make art a lifetime career. After they go into college, their interest cannot be stimulated by the courses of their majors. They cannot find their learning goals and motivation for selflearning. They fail to obtain a sense of accomplishment from academic studies, and eventually, fail to set up their own career plans. These are all important reasons for the "hollow heart disease" among college art students.

\subsubsection{The unique mindset of college art students}

Art students have a more innovative and divergent mindset. With stronger creativity and abstract thinking, they often transcend their original thinking modes. They tend to be skeptical and argumentative. In their own worlds where they learn and live, there exists two selves, the "ego" and the "true self." "Ego" refers to their social attributes, a self with conscious activities. Their consciousness can be both rational and emotional.

"True self" refers to the self who constructs artistic thinking, pursues ideals and achieves self-realization. Unlike ordinary students, the ego and the true self of art students tend to cause different worldviews inside them, with ego closer to real life and true self more inclined to perfectionism. For youths, developing their self-identity will become an important foundation for their maturity and success. If contradictions exist between these two different worldviews, the development of teenagers' worldview, outlook of life and values would be affected. The result is art students may feel confused, lonely, helpless in their study and life; they fail to find the value and meaning of being alive, causing serious effects on the mental health of art students. This also became one of the major causes of "hollow heart disease."

\subsubsection{Diverse Internet culture influences the values of art students}

With the rapid development of the new media, youths today are digital natives who almost live on the Internet. With the rise of We-Media, teenagers have been constantly influenced by different newly-born thoughts. In an information-overloaded society, without guidance of correct mainstream values, students might be overwhelmed by the substantial amount of information and lose the ability to distinguish right from wrong, resulting in unclear or lack of values and causing one of the major symptoms of "hollow heart disease" value nihilism. Due to the nature of arts and their own personality, art students tend to perceive and evaluate new things with innovative perspectives. They often have their own ways of understanding, daring to challenge a system, textbook knowledge, traditions or authorities. They are skeptical and argumentative. They pursue individualism, independence and a true self. With such psychological traits, when faced with the diverse cultures on the Internet, art students are more inclined to be eroded by the entertainment-oriented Internet. Gradually they will lose themselves in the tremendous amount of fragmented and biased information. They will lose their goals and directions, and walk right into the arms of “ hollow heart disease."

\section{EFFECTIVE WAYS TO TACKLE “ HOLLOW HEART DISEASE" AMONG COLLEGE ART STUDENTS}

\subsection{Construct a coordinated education mechanism of ideological and political courses with artistic characteristic}

Stereotypes that ideological and political courses are boring and close to brainwashing have been implanted in the mind of art students during their teenage years. When they get into college, they have lost all their interest in traditional ideological and political courses. Moreover, teaching methods like cramming cannot produce good learning effect. How to help students digest ideological and political knowledge has become a tough nut to crack in ideological and political courses. Therefore, the intensity and methods of ideological and political education shall be innovated to develop ideological and political education exclusively for art students. Under the “Three All-round Education" proposed by CPC' s General Secretary $\mathrm{Xi}$ Jinping, higher education institutions will shatter the divisions of professional education, management services and student ideological and political education, and integrate ideological and political education into all aspects of talent training, namely, the entire process of teaching, scientific research, management and services. With the problems and current conditions of art students in our mind, we should address the root cause and construct an ideological and political teaching system with artistic characteristics, combining vocational practices with ideological and political education, and integrating core socialist values throughout the entire process of education. Artworks with ideological and political meanings can be taken as the teaching materials, or the core socialist values can be chosen as the teaching guidance to show art students how to use Marxist worldview and methodology to observe, analyze, and solve problems. In these ways, we can change or even improve students' ideological level and ways of thinking, help them develop the correct worldview, outlook on life and values, and cultivate optimistic attitudes and peaceful minds inside them, with an aim to reduce or avoid the occurrence of "hollow heart disease." 


\subsection{Conduct mental health education with well- defined purposes}

As for mental health education, we should take improving students' mental health as the ultimate goal, with no limitation on forms or time. Throughout the four years in college, psychological problems are constantly arising and evolving. Therefore, mental health education should also be carried out to address the definite problems. We cannot just organize psychological assessments and relevant lectures in their freshman year, and then ignore their mental health problems later. Mental health education shall be planned and organized systematically based on the current stages they are in. Taking into consideration the characteristics of art students and talent training programs, we shall carry out targeted psychological education with various methods and focuses. For instance, based on the major characteristics and mental features of college art students, we can set up courses combining arts and psychology, such as the psychology of art, or organize related lectures. In this process, interactive and experiential teaching methods should be adopted as much as possible to stimulate students' curiosity and equip them with mental health related knowledge[4]. At the same time, class teachers shall set up a mental health ledger, and class members in charge of mental health shall keep realtime records of students' mental health by talking to class representatives, dormitory supervisors, and subject teachers, achieving full coverage of all the students' mental status. In this way, students with "hollow heart disease" can be discovered in a timely manner, and targeted psychological counseling and one-on-one follow-up guidance can be given to prevent the disease from progressing.

\subsection{Use new media to enrich students' campus spiritual life}

Campus and new media, as the main "venues" of college students' offline and online activities, play important roles in their mental health education. The ideological and political education in college should be

"changed through incidents, improved with the times and reformed in line with the trends", which means such education should be innovated in terms of its philosophy, methods and contents. Efforts should be made to combine arts with Internet culture, figure out what art students really want, and discover their mainstream interests, before we select the ways they are more willing to accept to conduct ideological and political education management. In terms of online channels, the teachers in charge of ideological and political education and other subjects should learn more about the Internet, familiarize themselves with online platforms such as TikTok, Bilibili, Weibo, and Qzone, and set up official accounts to communicate with students more closely, leading them to develop correct values. In the meantime, through established connections, teachers should gather relevant information and analyze the ideological and psychological features of art students. On the other hand, offline cultural activities that are conducive to value development, with core connotations and artistic exhibitions should be organized on campus. Eventually, a closed-loop for coordinated education system will be built with online guidance and offline participation, so as to promote the effective dissemination of mainstream values, provide guidance for college students to absorb the mainstream ideology and values and transform them into their own mental features and value appeals and ultimately, prevent " hollow heart disease" from happening on the students[5].

\section{CONCLUSIONS}

\subsection{Research objects and methods}

In this paper, art students in colleges and universities are taken as the research objects of mental health, and 826 art students are given questionnaires through questionnaire survey. According to the survey of valid samples, there are 204 people with " hollow heart disease" , accounting for $31 \%$ of the valid samples, which has become one of the most serious mental diseases of art students at present.

\subsection{Research significant results}

According to the data of questionnaire survey, eight pathological characteristics of hollow heart disease are summarized, which are depression, loneliness, selfdeficiency, external recognition, fear of being judged, self- hatred, suicidal thoughts and course of disease. This paper explains these eight characteristics one by one, and expounds the serious harm of "hollow heart disease" to the mental health education of art students. According to the characterization of "hollow heart disease", this paper deeply analyzes the causes of this disease among art students from four aspects: family factors, special academic structure, special thinking mode and the impact of multi-network culture.

\subsection{Feasibility promotion and prospect of research}

The mental health education for art students is different from other basic and professional courses. It should break the limitation of time and space, organically combine school, social education and family, follow the law of psychological development of art students, and carry out systematic, scientific and professional mental health guidance [6] . Based on the research on the representation, characteristics and causes of "hollow heart disease" of art college students, this paper puts forward the theoretical and practical methods to reduce the incidence of "hollow heart disease" from the 
aspects of teaching mode, mental health education of college students and campus cultural life. It provides data support for developmental education of art students in colleges and universities, and provides theoretical basis for further research and practice of mental health education for art students.

\section{REFERENCES}

[1] Wang Xinggang. Exploring Mental Disorders among College Students with "Hollow Heart Disease" [J]. Journal of Wuhan University of Technology, 2017(10):177-181. DOI https://kns.cnki.net/kcms/detail/detail.aspx?dbcode $=\mathrm{CJFD} \&$ dbname $=$ CJFDLAST2017 $\&$ filename $=\mathrm{WH}$ JT201705030\&v=P5c24vu8xA0XOOtG7hJruwCaq IraTpLArEi\%25mmd2Fdynahsm2xX3RICDWBW a9aqqJ6E0W

[2] Chen Jiadi. Analysis of Mental Health Education for Art Students in College [J]. Literary Education, 2012(7): 155-156.

DOI https://kns.cnki.net/kcms/detail/detail.aspx?dbcode $=$ CJFD \&dbname $=$ CJFD2012\&filename $=$ WXYZ20 $1207131 \& v=m B 6 d j F f Q z M q L 7 R b z d C 4 k k w 4 y 6 N d$ 6CtVrfXAsW\%25mmd2BVNA0P7D70Z8DyFxoc c\%25mmd2FYVMeMOv

[3] Liang Wenbo. Analysis of Mental Health Education for Art Students in College [J]. Education for Chinese After-school, 2011(4): 159. DOI : https://kns.cnki.net/kcms/detail/detail.aspx?dbcode $=$ CJFD $\&$ dbname $=$ CJFDLASN2019\&filename $=X$ WLL201108138\&v=hyENFeqgiNg3ASLfvskTo76 AiiHuPsoQRj\%25mmd2FlJQwwGYKGe\%25mmd 2Bx1U9LhlTEIXbnNWTT9

[4] Wang Qiong. Practices and Thoughts in the Psychoeducation for College Students [J]. Art Education, 2015(7): 131-132. DOI : https://kns.cnki.net/kcms/detail/detail.aspx?dbcode $=$ CJFD\&dbname $=$ CJFDLAST2015\&filename $=$ YSJ Y201507106\&v=N8NVF61UzFCpqJtu5ZkQ9enCy 1UGh8I\%25mmd2Bg6hoL5LGIIR8ncc55gqN1i\%2 5mmd2BYEx6IC1bW

[5] Wang Lei. Current Situation of Ideological and Political Education for College Students Under Internet's Influence [J]. Ideological and Political Studies, 2021(4): 3-4. DOI : https://kns.cnki.net/kcms/detail/detail.aspx?dbcode $=$ CJFD $\&$ dbname $=$ CJFDLAST2021 $\&$ filename $=J M$ SJ202104003\&v=7D1yBDXrEhPO301Jr6Rp2A8a NxE5ieosuzpRbHC4mUAesRXWnY0vJorm13Uv $\mathrm{BDEz}$

[6] Tian Wenhai, Liu Chunli. Mental Health Education and Ideological and Political Education for College
Students from the perspective of "Hollow Heart Disease" [J]. Modern Vocational Education, 2020(6): 22-23. DOI https://kns.cnki.net/kcms/detail/detail.aspx?dbcode $=$ CJFD $\&$ dbname $=$ CJFDLASN2020 $\&$ filename $=X D$ ZJ202026011\&v=yniTWQOwTmQ1\%25mmd2BJI qFxD70H5v466K\%25mmd2FF2GdhmpIkEXIRX1 9nNii4ueO0xMMBgtJu7o 